Koichi Ozaki • Tsutomu Fujiwara • Yusuke Nakamura

Ei-ichi Takahashi

\title{
Isolation and mapping of a novel human kidney- and liver-specific gene homologous to the bacterial acetyltransferases
}

Received: April 27, 1998 / Accepted: June 17, 1998

\begin{abstract}
Using the differential display method to detect tissue-specific genes, we isolated a novel human gene, designated TSC501, that is expressed in kidney and liver. The cDNA contained an open reading frame of 681 nucleotides encoding 227 amino acids. The predicted product showed homologies in amino acid sequence to three different bacterial acetyltransferases, enzymes that are involved in drug resistance. Radiation hybrid mapping localized this gene to human chromosome bands 2p12-p13.1.
\end{abstract}

Key words Tissue-specific gene · "Knock-out" mouse Differential display · Acetyltransferase - Drug resistance

\section{Introduction}

Human genome analyses, particulaly large-scale cDNA sequencing efforts, have disclosed thousands of novel genes. Examining the biochemical and physiological functions of the products of each of these genes has become an important issue. Although gene targeting is an excellent method for investigating physiological function in vivo, producing a "knock-out" mouse for each new gene is very laborious; moreover, targeting of certain types of genes, particularly housekeeping genes, can be expected to result in embryonic lethality. On the basis of our assumption that it should be possible to disrupt, without embryonic lethality, genes that are expressed only in specific adult tissues, we have focused

K. Ozaki $(\bowtie) \cdot$ T. Fujiwara $\cdot$ E. Takahashi

Otsuka GEN Research Institute Otsuka Pharmaceutical Co., Ltd., 463-

10 Kagasuno Kawauchi-cho Tokushima 771-0192, Japan

Tel. +81-886-65-2888; Fax +81-886-37-1035

e-mail: ozakik@otsuka.genome.ad.jp

Y. Nakamura

Laboratory of Molecular Medicine, Human Genome Center, Institute of Medical Science, The University of Tokyo, Tokyo 108-0071, Japan The nucleotide sequence data reported in this paper will appear in the DDBJ/EMBL/GenBank databases with the accession number AB013094. our efforts on isolating tissue-specific genes that would be appropriate for gene-targeting experiments.

Aminoglycoside-aminocyclitol antibiotics form a large family of antimicrobial agents, including streptmycin, gentamicin, kanamycin, and amikacin, which act principally against gram-negative bacteria (Davis, 1986). Plasmidencoded resistance to these agents is mediated by enzymatic modification; i. e., by acetylation of amino groups or by adenylation or phosphorylation of hydroxyl residues (Haas and Dowding 1975; Mitsuhashi, 1982).

Differential display (Liang and Pardee 1992; Liang et al. 1992; Bauer et al. 1993; Utans et al. 1994; Donohue et al. $1995)$ is an effective method for isolating genes that are expressed differentialy among separate tissues. We previously isolated several human testis-specific genes in this way, and demonstrated the power of differential display to do this even when the genes were expressed at levels as low as 1 in a million molecules of total mRNA (Ozaki et al. 1996).

In the work reported here, this approach permitted us to isolate a novel kidney- and liver-specific gene homologous to the bacterial acetyltransferases.

\section{Materials and methods}

Differential display

The differential display procedure was performed essentially as described by Liang and Pardee (1992). Subcloning of amplified cDNA fragments was described previously (Ozaki et al. 1996). Nucleotide sequences were determined by means of an ABI 377 auto-sequencer (Applied Biosystems, Foster City, CA, USA).

Northern-Blot analysis

Human Multiple-Tissue Northern (MTN) blots I and II (Clontech, Palo Alto, CA, USA) were probed with a cDNA 
fragment (TSC501), obtained from the differential display, which had been labeled with $\alpha-\left[{ }^{32} \mathrm{P}\right]$ deoxycytidine triphosphate (dCTP) with a random primed DNA labelling kit (Boehringer manmheim, Tokyo, Japan). The membranes were pre-hybridized and then hybridized according to the manufacturer's protocol. Washed membranes were autoradiographed for $16 \mathrm{~h}$ at $-80^{\circ} \mathrm{C}$.

\section{Screening of cDNA}

A human normal kidney cDNA library was constructed using oligo(dT)-primed normal human lung cDNA and Umi-ZAP XR vector (Stratagene, La Jola, CA, USA). In total, $1 \times 10^{6}$ clones were screened with the $\alpha-\left[{ }^{32} \mathrm{P}\right] \mathrm{dCTP}$ labeled cDNA fragment (TSC501). Positive clones were selected and their insert DNAs were excised in vivo in pBluescript II $\mathrm{SK}(-)$ according to the supplier's recommendation.

\section{Radiation-Hybrid mapping}

Using polymerase chain reaction (PCR) primers generated from the 3'-UTR of the TSC501 cDNA, we tested the amplified product against the Genbridge- 4 whole-genome ra- diation hybrid panel (Research Genetics, Huntsville, AL, USA) (Walter et al. 1994). The primer sequences were as follows: forward,CTT TCT GTG TGT ATT GGT CAG; reverse,TGG ACA ATA AGC TTT ATT GC. Amplification results were submitted to the Radiation Hybrid Mapper server at the Whitehead Institute/MIT Center for Genome Research (http://www-genome.wi.mit.edu/cgi-bin/ contig/rhmapper.pl).

\section{Results and discussion}

\section{Differential display}

To identify human genes expressed in a tissue-specific manner, we compared differential display patterns using mRNAs isolated from brain, liver, lung, stomach, pancreas, spleen, heart, skeletal muscle, breast, visceral fat, prostate, and kidney. With one primer combination we identified a PCR product that was specifically seen in kidney and liver (Fig. 1a). This product, TSC501, was cloned and sequenced; it consisted of 116 nucleotides. Comparison of the nucleotide data with DNA sequences in the GenBank and European Molecular Biology Laboratory (Heidelberg) (EMBL)
Fig. 1 a Differential display patterns of various human tissues. Arrows indicate specific bands (TSC501) among 12 tissues. b Northern blotting of TSC501 in various adult human tissues. The expression pattern was examined using as a probe the cDNA fragment isolated by differential display. $P B L$, Peripheral blood lymphocytes; $s k$, skeletal; int, intestine a

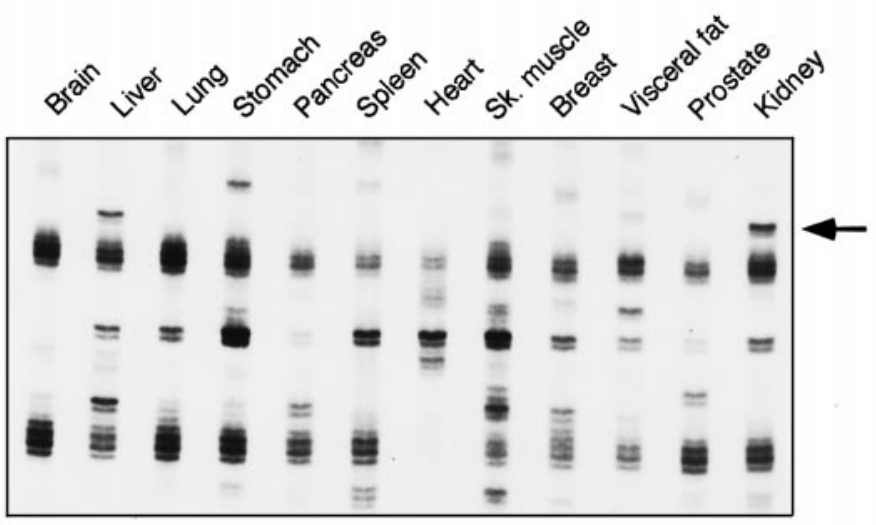

b
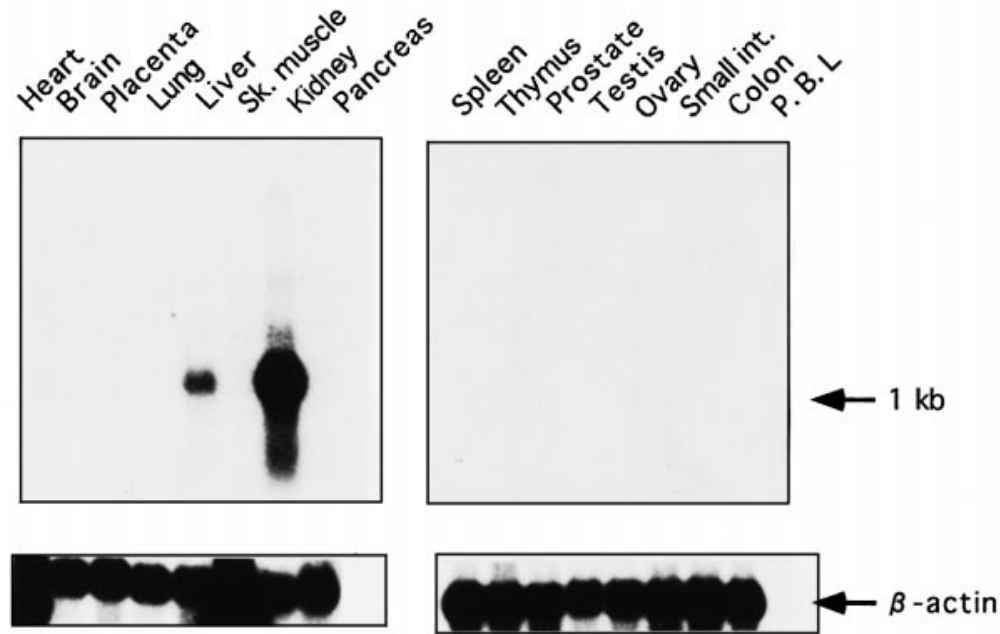
databases (Releases 102.0 and 51.0, respectively) using the FASTA program (Person and Lipman 1988), revealed no significant homology of this PCR product with any archived DNA sequence. To confirm the expression pattern observed in the differential-display profile, we examined the expression of TSC501 cDNA in various human adult tissues by Northern-blot analysis. As shown in Fig. 1b, a 1$\mathrm{kb}$ transcript corresponding to TSC501 was observed specifically in the kidney and liver.

Cloning, sequencing of full-length TSC501 cDNA, and mapping of the TSC501 gene

A human kidney cDNA library $\left(1 \times 10^{6}\right.$ plaques $)$ was screened, using the cDNA fragment isolated through the differential display experiment as the probe. We identified about 500 positive clones corresponding to TSC501. On the basis of this result, we estimated the abundance of transcripts among total kidney mRNAs to be $0.05 \%$.

The assembled cDNA sequence contained 960 nucleotides (GenBank accession number, AB013094) encoding 227 amino acids with a calculated molecular weight of 25,617 Daltons. The amino acid sequence encoded by TSC501 contained a possible hydrophobic signal motif near the $\mathrm{N}$ terminus $\left(\mathrm{Leu}^{55}-\mathrm{Ala}^{78}\right.$ ) and one possible transmembrane domain near the $\mathrm{C}$ terminus $\left(\mathrm{Gln}^{198}-\mathrm{Pro}^{220}\right)$. A comparison with known proteins in the public database, using the FASTA program, identified $27 \%, 26 \%$, and $29 \%$ identities, respectively, to Streptomyces lavendulae Sta T (Horinouchi et al. 1987), Escherichia coli RimI (Yoshikawa et al. 1987), and E. coli aacC1 (Wohlleben et al. 1989) (Fig. 2). Sta $\mathrm{T}$ and aacC1 are enzymes that transfer an acetyl group from acetyl coenzyme A to the beta amino-group of the beta-lysine moiety of streptothricin and gentamicin, respectively; RimI acetylates the N-terminus of the ribosomal S-18 protein. In the human, several acetyltransferases are implicated in drug metabolism. For example, deficient function of the enzyme arylamine $\mathrm{N}$-acetyltransferase in the liver causes impaired metabolism of many therapeutically useful arylamine and hydrazine drugs (Weber and Hein 1985). These include, among many others, the antituberculosis drug isoniazid, the chemotherapeutic agent dapsone, the widely used antiarrhythmic procainamide, and sulfamethazine, as well as many other sulfonamides (Weber 1987). Important toxicological consequences may arise, because, in addition to drugs, a number of potential carcinogens, including B-naphthylamine, benzidine, and 2-aminofluorene, are substrates of liver arylamine $\mathrm{N}$ acetyltransferase. Furthermore, a large body of clinical data suggests associations between acetylator phenotype and spontaneous or drug-induced disease such as bladder cancer (Evans 1984), Gilbert's disease (Platzer et al. 1987), and leprosy (Ellard et al. 1972). The structural similarities suggest that the TSC501 product could function as an acetyltransferase, and if so, that it might mediate drug metabolism in kidney and liver.

The TSC501 gene was localized to bands p12-p13.1 of chromosome 2 by radiation hybrid mapping, through linkage to the MIT framework marker WI-10809 (lod > 3).

Although the function of the TSC501 protein is unknown, its similarity to acetyltransferase and its specific and abundant expression in normal kidney and liver suggest that it plays an important role in the development and maintenance of normal kidney and liver structure and function.

Acknowledgements We thank Yuka Fujii and Katsue Yamada for technical assistance, Atsushi Kawai for sequencing, and Hiroichi Shinomiya for RH mapping.

This work was supported in part by grants from the Ministry of Education, Culture, Sports and Science of Japan.

\section{References}

Bauer D, Muller H, Reich J, Riedel H, Ahrenkiel V, Warthoe P, Strauss M (1993) Identification of differentially expressed mRNA species by an improved display technique (DDRT-PCR). Nucleic Acids Res 21: 4272-4280



Fig. 2 Alignment of the TSC501 protein with three different bacterial acetyltransferases. Residues identical to TSC501 are marked in black. Homologous residues are marked in gray 
Davis J (1986) A new look at antibiotic resistance. FEMS Microbiol Rev 39: 363-371

Donohue PJ, Allberts GF, Guo Y, Winkles JA (1995) Identification by targeted differential display of an immediate early gene encoding a putative serine/threonine kinase. J Biol Chem 270: 10351-10357

Ellard GA, Gammon PT, Helmy HS, Rees RJW (1972) Dapsone acetylation and the treatment of leprosy. Nature 239: 159-160

Evans DAP (1984) Survey of the human acetylator polymorphism in spontaneous disorders. J Med Genet 21: 243-253

Haas MJ, Dowding JE (1975) Aminoglycoside modifying enzyme. Methods Enzymol 43: 611-640

Horinouchi S, Furuya K, Nishiyama M, Suzuki H, Beppu T (1987) Nucleotide sequence of the streptothricin acetyltransferase gene from Streptomyces lavendulae and its expression in heterologous hosts. J Bacteriol 169: 1929-1937

Liang P, Pardee AB (1992) Differential display of eukaryotic messenger RNA by means of the polymerase chain reaction. Science 257: 967-971

Liang P, Averboukh L, Pardee AB (1993) Distribution and cloning of eukaryotic mRNAs by means of differential display: Refinements and optimization. Nucleic Acids Res 21: 3269-3275

Mitsuhashi S (1982) Antibacterial activity of aminoglycoside antibiotics. Springer-Verlag, Berlin

Ozaki K, Kuroki T, Hayashi S, Nakamura Y (1996) Isolation of three testis-specific genes (TSA303, TSA806, TSA903) by a differential mRNA display method. Genomics 36: 316-319
Person WR, Lipman DJ (1988) Improved tools for biological sequence comparison. Proc Natl Acad Sci USA 85: 2444-2448

Platzer R, Kupfer A, Bircher J, Preisig R (1987) Polymorphic acetylation and aminopyrine demethylation in Gilbert's syndrome. Eur $\mathrm{J}$ Clin Invest 8: 219-223

Utans U, Liang P, Wyner LR, Karnovsky MJ, Russell ME (1994) Chronic cardiac rejection: Identification of five upregulated genes in transplanted hearts by differential mRNA display. Proc Natl Acad Sci USA 91: 6463-6467

Walter MA, Spillett DJ, Thomas P, Weissenbach J, Goodfellow PN (1994) A method for constructing radiation hybrid maps of whole genomes. Nat Genet 7: 22-28

Weber WW (1987) The acetylator genes and drug response. Oxford University Press, New York

Weber WW, Hein DW (1985) N-Acetylation pharmacogenetics. Pharmacol Rev 37: 25-79

Wohlleben W, Arnold W, Bissonnette L, Pelletier A, Tanguay A, Roy PH, Gamboa GC, Barry GF, Aubert E, Davies J (1989) On the evolution of Tn21-like multiresistance transposons: sequence analysis of the gene $(\mathrm{aacC} 1)$ for gentamicin acetyltransferase-3-I (AAC(3)-I), another member of the Tn21-based expression cassette: Mol Gen Genet 217: 202-208

Yoshikawa A, Isono S, Sheback A, Isono K (1987) Cloning and nucleotide sequencing of the genes rim I and rim $\mathrm{J}$ which encode emzymes acetylating ribosomal proteins S18 and S5 of Escherichia coli K12: Mol Gen Genet 209: 481-488 